\title{
Adherence to Post-polypectomy Guidelines: Just One Piece of an Important Puzzle
}

\author{
Audrey H. Calderwood ${ }^{1}$
}

Published online: 25 June 2015

(c) Springer Science+Business Media New York 2015

Adherence to post-polypectomy surveillance guidelines not only is an essential element of high-quality colorectal cancer (CRC) prevention programs, but also offers novel and important areas for further investigation. The growing emergence of accountable care organizations (ACOs), which care for individuals using finite resources, further emphasizes the importance of adherence to surveillance guidelines, which aim to prevent the development of advanced adenomas and CRC, while limiting unnecessary risk from exposure to too frequent procedures. Furthermore, overuse of surveillance colonoscopy services adversely impacts the capacity to perform colonoscopies, increasing the opportunity cost for those who require initial screening colonoscopy.

The importance of appropriate use of surveillance colonoscopy is highlighted by the development, endorsement, and inclusion of Quality Measure \#185 (Endoscopy/ Polyp Surveillance: Colonoscopy Interval for Patients with History of Adenomatous Polyps-Avoidance of Inappropriate Use) in Medicare's Physician Quality Reporting System [1], a "pay-for-reporting" program that provides incentives and payment adjustments for satisfactorily reporting quality measures. Under this program, physicians who do not satisfactorily report surveillance colonoscopy data during the 2015 performance year will receive a financial penalty of $-2.0 \%$ in 2017 [1].

The existing literature has robustly documented the underuse and overuse of surveillance colonoscopy among

Audrey H. Calderwood

audrey.calderwood@bmc.org

1 Section of Gastroenterology, Department of Medicine, Boston Medical Center, 85 East Concord Street, Room 7724, Boston, MA 02118, USA different populations within and outside the USA [2, 3]. Several studies have identified predictors of physicians' non-adherence to guideline recommendations. With regard to the timing of subsequent surveillance colonoscopy, Saini et al. [4] reported that the lack of knowledge of specific surveillance intervals stated in the guidelines and disagreement with the guidelines was associated with guideline-discordant recommendations. Iskandar et al. [5] identified that physicians who were trained before 1990, were currently in training, performed a low volume of colonoscopies, or practiced at a non-academic center were more likely to make guideline-discordant recommendations. Both studies surveyed guideline knowledge eliciting recommendations based on hypothetical scenarios, but did explore the relationship between survey responses and actual clinical practice.

In this issue of Digestive Diseases and Sciences, Patel et al. [6] used a combination of methods to address reasons for physicians' deviation from post-polypectomy surveillance colonoscopy guidelines. The study included an online survey of gastroenterologists and trainees within a single university system. They also analyzed a chart review of 600 patients under the supervision of the surveyed gastroenterologists, seeking to delineate the predictors of guideline concordance and guideline discordance. Among the analyzed patients, the overall rate of guideline-appropriate recommendations was $85 \% ; 13 \%$ of patients were recommended a shorter surveillance interval than indicated by guidelines, and only $3 \%$ of the patients were recommended a longer surveillance interval than indicated by guidelines. Guideline knowledge and trainee status were associated with superior guideline adherence, with trainees 2.5-fold more likely to adhere with guidelines, in contrast to the findings of Iskandar et al. [5]. Conversely, uncertainty among physicians regarding the aggressiveness of 
guidelines was associated with a $40 \%$ decrease in the likelihood of guideline adherence. The authors should be commended for linking survey data from individual physicians with actual recommendations made as part of clinical care.

The results from the hypothetical clinical scenarios used in the survey are interesting: In a scenario addressing piecemeal resection of advanced adenomas, $39 \%$ of respondents chose longer-than-recommended surveillance intervals, of concern since it is estimated that $19-27 \%$ of interval cancers arise in the same region of the colon where prior polyps were removed [7]. Residual polyp tissue from prior incomplete resection might be the source of interval cancer in these cases rather than a missed or de novo lesion. If this study by Patel et al. [6] is generalizable to other practice settings, the results should stress how incomplete polypectomy contributes to the formation of interval CRC, and signal the need for appropriate, targeted training in this regard, whether through continuing medical education provided by the gastroenterology societies or through other avenues. For a scenario including the management of high-risk adenomas, $64 \%$ of respondents overestimated the rate of development of metachronous high-risk adenomas among high-risk patients. Moreover, $46 \%$ chose a shorter surveillance interval than recommended by guidelines. In an attempt to understand the motivation underlying the scenario responses, Patel et al. [6] reported that $82 \%$ of respondents cited fear of missed cancer and $36 \%$ cited malpractice concerns. Missing cancers is perhaps the greatest fear of gastroenterologists involved in screening and surveillance colonoscopy due to the perception that CRC is a largely preventable disease, yet there is a well-documented, finite, baseline polyp miss rate [7]. Placing more emphasis on high-quality colonoscopic examinations may help bolster individual physician and group confidence in the sensitivity of the procedure for detecting neoplasia, thereby by shifting behavior to more guideline-appropriate surveillance intervals. Quality metrics such as bowel preparation quality, cecal intubation and adenoma detection rates, and to a lesser extent withdrawal time, will help to standardize practice and promote trust in colonoscopy as a procedural service.

While fear of missed cancer was identified as the primary reason impacting surveillance interval recommendations in the survey, it was not associated with guideline adherence in the reviewed patient charts. Rather, clinical practice was affected mostly by guideline knowledge and trainee status, providing hope since it is easier to increase guideline knowledge than to change one's fear of missing cancer. Yet, broad dissemination of guideline knowledge has been difficult, with an estimated lag time between guideline publication and adoption of $>10$ years [8]. Our professional societies may need to draw from other areas of medicine or even other disciplines to determine how to best disseminate guidelines knowledge.

One limitation inherent to any survey methodology is whether physicians' responses to the survey were candid, truly reflecting their beliefs and practices. In particular, malpractice concerns and financial incentives were both cited as a motivating factor for surveillance guideline recommendations, but by only a minority of respondents, the percentage of which may have been skewed by the large portion of trainees (57\%) in the survey who are largely shielded from these concerns while in training. Since the contributions of malpractice concerns and financial incentives likely depend on the practice setting, it would be interesting to evaluate their influence in other settings beyond academia, including community-based private practices.

Another striking finding was that $22 \%$ of the reviewed charts were missing a final recommendation for a surveillance interval. Although this percentage is concerning, it is not surprising given the extensive coordination of data and communication that is required in managing postpolypectomy surveillance interval recommendations. A delay between the colonoscopy and the final surveillance interval recommendation may occur while the physician awaits the pathology results, integrates the pathology with the colonoscopy findings, and finally communicates a recommendation to the patient and referring provider. Although recommendations may have been made but not documented in this study, documentation is a key to success in the delivery of surveillance colonoscopy care due to the long intervals of time between examinations (i.e., $3-5$ years).

While appropriate adherence to surveillance colonoscopy begins with a recommendation from the performing endoscopist, perhaps an even more clinically relevant outcome is whether patients actually undergo surveillance colonoscopy in a timely, guideline-concordant manner. This somewhat de-emphasizes the importance of the physician's recommendations, highlighting the difference between process and outcome measures. In other words, the physician recommendation is just one, albeit important, component to achieving the overall goal of performing colonoscopic surveillance at guideline-specified intervals (Table 1). Taking it one step further, the ultimate outcome, though less feasible to measure, is whether CRCs are prevented among those with a history of adenomas.

Several factors that extend beyond physicians' recommendations when evaluating patient adherence with guideline-concordant surveillance colonoscopy intervals include: communication with primary care providers, patient preferences, and competing medical issues, to name a few. Brueckl et al. [9] reported reasons for non-adherence with surveillance colonoscopy among patients with a 
Table 1 Patient-, provider-, and system-level factors associated with inappropriate use of surveillance colonoscopy

\begin{tabular}{ll}
\hline Level & Factors associated with appropriate use of surveillance colonoscopy \\
\hline Patient & Demographic characteristics: older age, female gender \\
& Engagement in primary care: lower number of visits, having co-morbidity \\
& Beliefs: fear, feeling asymptomatic, lack of recall or knowledge \\
& Demographic characteristics: career age, trainee status \\
Provider & Practice characteristics: setting, geographic location, volume of procedures \\
& Knowledge of guidelines \\
& Disagreement with guideline aggressiveness \\
& Communication between endoscopist and primary care provider \\
System & Use of electronic health record \\
& Alert system \\
\hline
\end{tabular}

history of large $(>1 \mathrm{~cm})$ adenomas, identifying lack of knowledge about surveillance intervals, lack of symptoms, and fear of the examination as the main factors. Furthermore, female gender and older age were associated with non-adherence. By evaluating predictors of surveillance colonoscopy adherence within an open-access safety net system in my own work, I reported that only $40 \%$ of patients returned for surveillance colonoscopy at the recommended interval [10]. Engagement in primary care as measured by the number of primary care visits and having "adenoma" listed on the problem list of the electronic health record (EHR) at the referring provider's practice was associated with greater likelihood of adherence.

With regard to logistics, should the patient be responsible for remembering when the procedure is scheduled and for calling for an appointment? This seems unreasonable and likely to fail. Should the endoscopist maintain a database and recall patients directly when surveillance is due? Or should the primary care provider have responsibility for coordinating with the patient? Endoscopists, primary care providers, and the systems in which we work will all need to facilitate the logistics of arranging for surveillance colonoscopy in order to ensure the best care for our patients. With changing models of care including the emergence of ACOs, we will all be held accountable.

In the future, online-based updated centralized decision support systems for delivering recommendations for the timing of repeat procedures will be valuable in an everchanging clinical field. Further, the use of system-based interventions to promote on-time colonoscopy (e.g., physician alerts and reminder letters) will be facilitated within the EHR, and even more so by integrated EHRs that communicate across different systems of care. Appropriate post-polypectomy surveillance begins with a physician's recommendation and continues with on-time patient follow-up at colonoscopy. Let us put the first piece of this puzzle in the right spot as an initial step toward achieving an overall solution.
Acknowledgments Audrey Calderwood is supported by the NIH K08 DK090150-05 and a Boston University School of Medicine Department of Medicine Career Investment Award.

\section{References}

1. Medicare.gov: Physician Quality Reporting System (PQRS). http://www.cms.gov/Medicare/Quality-Initiatives-Patient-Assess ment-Instruments/PQRS/. Accessed 22 May 2015.

2. Schoen RE, Pinsky PF, Weissfeld JL, et al. Utilization of surveillance colonoscopy in community practice. Gastroenterology. 2010;138:73-81.

3. van Heijningen EM, Lansdorp-Vogelaar I, Steyerberg EW, et al. Adherence to surveillance guidelines after removal of colorectal adenomas: a large, community-based study. Gut. 2015. doi:10. 1136/gutjnl-2013-306453.

4. Saini SD, Nayak RS, Kuhn L, Schoenfeld P. Why don't gastroenterologists follow colon polyp surveillance guidelines?: results of a national survey. J Clin Gastroenterol. 2009;43: $554-558$.

5. Iskandar H, Yan Y, Elwing J, Early D, Colditz GA, Wang JS. Predictors of poor adherence of US gastroenterologists with colonoscopy screening and surveillance guidelines. Dig Dis Sci. 2015;60:971-978.

6. Patel N, Tong L, Ahn C, Singal AG, Gupta S. Post-polypectomy guideline adherence: importance of belief in guidelines, not guideline knowledge or fear of missed cancer. Dig Dis Sci. (Epub ahead of print). doi:10.1007/s10620-015-3685-x.

7. Lieberman DA, Rex DK, Winawer SJ, et al. Guidelines for colonoscopy surveillance after screening and polypectomy: a consensus update by the US Multi-Society Task Force on Colorectal Cancer. Gastroenterology. 2012;143:844-857.

8. Grol R. Successes and failures in the implementation of evidencebased guidelines for clinical practice. Med Care. 2001;39:II46II54.

9. Brueckl WM, Fritsche B, Seifert B, et al. Non-compliance in surveillance for patients with previous resection of large $(>$ or $=1 \mathrm{~cm}$ ) colorectal adenomas. World $J$ Gastroenterol. 2006;12:7313-7318.

10. Calderwood AH, Schroy PC, Cabral H, Burgess J. Predictors of adherence to post-polypectomy surveillance. Gastroenterology. 2014;146:S-547. 